\title{
DISEÑO DE UN MODELO DE CONSULTORIO EMPRESARIAL PARA LA UNAD ECACEN ZONA OCCIDENTE
}

\section{A BUSINESS CONSULTING MODEL DESIGN FOR THE UNAD ECACEN ZONA OCCIDENTE}

Autor 1 - Víctor Daniel Bedoya García Licenciado en inglés como Lengua Extranjera UNAD, Especialista en Educación Superior a Distancia UNAD, estudiante de Maestría en Administración de Organizaciones UNAD y perteneciente al grupo de investigación CANANGUCHALES (Código COL0060156), y al semillero de investigación SIDESGOP.

ORCID 0000-0002-4266-4580

email.vdbedoyag@unadvirtual.edu.co

Autor 2 - Nelson Augusto Serna Porras

Maestrando en Administración de Organizaciones, Administrador de Empresas. Docente ocasional de la UNAD, y perteneciente al grupo de investigación CANANGUCHALES (Código COL0060156), y al semillero de investigación SIDESGOP.

ORCID 0000-0002-4575-4645

email.nelson.serna@unad.edu.co

Autor 3 - Pablo Fernando Sánchez Osorio

Especialista en Alta Gerencia, Ingeniero industrial. Docente ocasional de la UNAD, y perteneciente al grupo de investigación CANANGUCHALES (Código COL0060156), y al semillero de investigación SIDESGOP.

ORCID 0000-0003-1682-4584

email.pablo.sanchez@unad.edu.co

\section{RESUMEN.}

El presente trabajo tiene por objeto diseñar un modelo de consultorio empresarial en la UNAD ECACEN ZOCC, de modo que dinamice las relaciones por parte de la Universidad y el sector productivo, y que genere, a su vez, mayores posibilidades de inserción laboral de sus egresados. Tres aspectos están asociados a la concreción del objeto principal: a) la determinación de la pertinencia frente al diseño de un consultorio empresarial para los estudiantes y egresados, la escuela ECACEN y el sector productivo; b) la propuesta de un portafolio de servicios a partir de las líneas estratégicas y los tipos de servicios que se requieren para ofrecer el consultorio empresarial 
de la UNAD Zona Occidente; c) el diseño de un modelo de consultorio administrativo, financiero y contable para la ECACEN ZOCC que vincule aspectos estratégicos, organizacionales, administrativos, financieros, logísticos y operativos.

El estudio inició con un procedimiento de revisión sistemática de fuentes documentales, que favorecieron la contextualización de la dinámica asociada a la creación e implementación de consultorios empresariales y su capacidad para facilitar el ingreso al mercado laboral en beneficio de los egresados de las IES, posteriormente, se tomaron a los resultados de una investigación realizada durante el año 2020 por el Semillero de Investigación en Desarrollo Sostenible, Gestión Organizacional y Prospectiva (SIDESGOP), perteneciente al grupo de Investigación CANANGUCHALES, desde el cual se realizó una encuesta (previamente validada por pares) a la comunidad de egresados. Se realizará una investigación descriptiva, con enfoque mixto.

Palabras Clave: Consultorio empresarial, egresados universitarios, empresas, empleo, relación.

\section{ABSTRACT.}

The purpose of this work is to design a business consulting model for the UNAD ECACEN ZOCC, so that, the University and the productive sector develop a more dynamic relationship, which, in turn, generate greater employment possibilities for its graduates. Three aspects are associated with the realization of the main object: a) the pertinence determination corresponding the design of a business consulting office for students and graduates, the ECACEN school and the productive sector; b) a portfolio proposal of services based on the strategic lines and the types of services required to be offered; $c$ ) the design of an administrative, financial and accounting consulting model for the UNAD ECACEN ZOCC that links strategic, organizational, administrative, financial, logistical and operational aspects.

The study began with a systematic review of documentary sources, which facilitated the contextualization of the dynamics associated with the creation and implementation of business consultancies and their capacity to boost the influx of graduates into the labor market. The results of a research conducted in 2020 by the Semillero de Investigación en Desarrollo Sostenible, Gestión Organizacional and Prospectiva (SIDESGOP) were subsequently used, this group belongs to the investigation research team so called CANANGUCHALES. A survey (previously validated by peers) was 
applied among the alumni community. A descriptive research with a mixed approach was conducted.

Keywords: Business consulting, university graduates, companies, employment, relationship.

\section{INTRODUCCIÓN.}

Varias son las problemáticas que enfrentan los egresados de las instituciones de educación superior (IES) colombianas. Su acceso al campo laboral es, sin duda, un asunto evidente y complejo. Dentro de los aspectos que complejizan la articulación de los egresados de las IES colombianas en el mercado laboral, se pueden destacar: el incremento del acceso a la educación superior por parte de la población en general que crea una mayor competencia para acceder a un empleo, el auge de las nuevas tecnologías de la información y comunicación (TIC), los cambios sociales, económicos y culturales que ha traído consigo la pandemia generada por el COVID-19, y muchas otras situaciones que se ven probadas con "la incapacidad del mismo mercado laboral para absorber a los nuevos titulados" (Rus \& Gragmena, 2018).

Ahora, las IES como actores corresponsables no deberían mostrarse impávidas ante el camino de los titulados. Las IES deben establecer procesos de proyección social como parte de su responsabilidad en torno a sus estudiantes y colaboradores (Benavidez y Villanueva, 2019). Actualmente, se presenta un panorama de baja empleabilidad a nivel nacional (DANE, 2020), que también afecta a los graduados universitarios. Es así como la credibilidad y reputación de las IES puede depender, en parte, de las gestiones que las estas realicen respecto a las estrategias para favorecer el acceso de sus graduados a puestos de trabajo.

Por lo tanto, basado en los resultados obtenidos de un estudio previo, se avista la necesidad de crear mecanismos que favorezcan el ingreso de los egresados de las IES al mercado laboral y, asimismo, que fortalezcan la relación entre las IES con el sector empresarial. Así las cosas, el diseño de un modelo de consultorio, administrativo, financiero y contable para la ECACEN ZOCC que vincule aspectos estratégicos, organizacionales, administrativos, financieros, logísticos y operativos, puede ser positivo para la UNAD ECACEN ZOCC, vislumbrando una eventual implementación, en consideración de varias perspectivas: a) se generan mayores y mejores posibilidades de inserción laboral de sus egresados, b) se dinamizan las 
relaciones entre la Universidad y el sector empresarial, c) se corresponde a la inversión y confianza de los egresados.

\section{METODOLOGÍA.}

El estudio empezó con la consulta de fuentes bibliográficas secundarias como insumo de información para contextualizar sobre los consultorios empresariales y su capacidad para facilitar el ingreso de los egresados de las IES al mercado laboral. Se realizará una investigación descriptiva con enfoque mixto. Se utilizará el diseño de triangulación concurrente, -DITRIAC(Hernández et al., 2014), para la recolección, análisis e interpretación cruzada de datos. En este mismo sentido, correspondiente al proceso cuantitativo, con la base de un estudio previo, se realizará un muestreo aleatorio simple sin reemplazo sobre base de datos de los estudiantes a quienes, en su momento, les falta menos de catorce (14) créditos académicos para culminar cualquiera de los programas ofertados en la UNAD en la ECACEN ZOCC. Se les suministrará un cuestionario para recoger y caracterizar (entre otras cosas) sus necesidades de empleo. Se les consultará a los estudiantes con preguntas cerradas sobre los asuntos más críticos al momento de buscar una ubicación laboral como profesionales en su futuro próximo. Ahora, frente a la parte cualitativa, se realizarán dos (2) grupos de enfoque para consultar a empresarios (específicamente, a aquellos vinculados con negocios en la Central Mayorista de Antioquia), docentes y líderes de programa sobre la percepción general relativa a los problemas que enfrentan los egresados de las IES para lograr una ubicación laboral alineada con sus profesiones.

Así las cosas, se ejercerá una comparación de los datos obtenidos en los dos procesos - cuantitativo vs cualitativo - para determinar la viabilidad de implementar un consultorio empresarial en la ECACEN ZOCC, de modo que se proceda, inicialmente, con el diseño de un modelo $y$, eventualmente, se aplique una prueba piloto en una empresa que requiera del acompañamiento que conlleve al fortalecimiento de sus áreas funcionales (Montoya et. al, 2018). Finalmente, se elaborará un informe con los resultados finales logrados. La investigación tendrá una duración menor o igual a cuatro meses después de haber sido iniciada (Daros, 2002).

\section{DISCUSIÓN Y RESULTADOS PARCIALES.}

Como fue mencionado previamente, se realizó una búsqueda de información más localizada en la Escuela de Ciencias Administrativas, Contables, Económicas y de Negocios (ECACEN) de la Universidad Nacional Abierta y a 


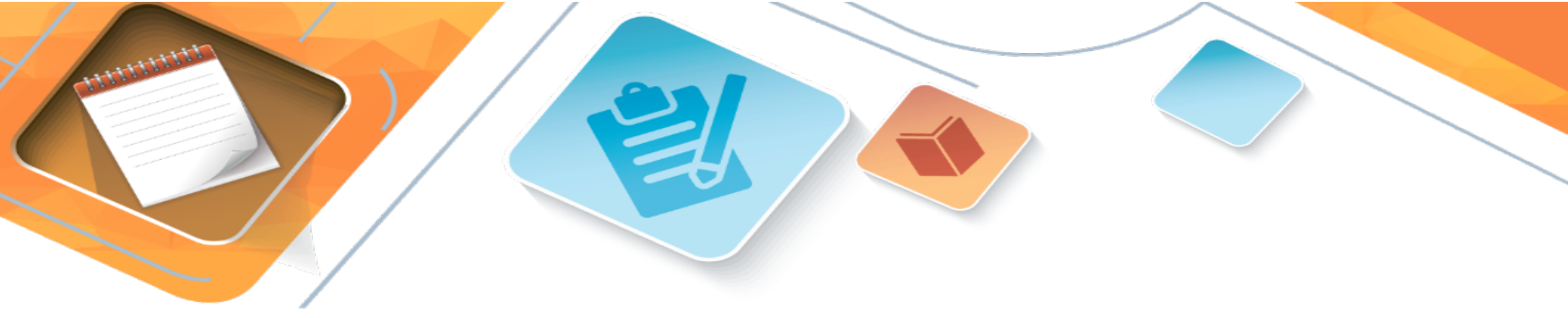

Distancia (UNAD), específicamente en la Zona Occidente (ZOCC). Mediante solicitud escrita fue posible acceder a los resultados de una investigación realizada durante el año 2020 por el Semillero de Investigación en Desarrollo Sostenible, Gestión Organizacional y Prospectiva (SIDESGOP), perteneciente al grupo de Investigación CANANGUCHALES, desde el cual se realizó una encuesta (previamente validada por pares) a la comunidad de egresados.

El instrumento que fue lanzado a un total de 1.164 egresados en el mes de noviembre y obtuvo un total de 324 respuestas, tenía como fin era la recolección de información para la construcción de un mapa de conocimiento conformado por los factores que intervienen en la relación existente entre los egresados de la UNAD y el mercado laboral. Se obtuvo información valiosa sobre la opinión de los egresados en relación con el tema de estudio de la presente propuesta de investigación. Los resultados mencionados fueron trasladados a un diagrama de Ishikawa (espina de pescado) con el fin de obtener una mayor claridad, tal como se observa en la figura 1.

Los datos consultados dan cuenta de un $78,1 \%$ de los graduados que consideran una insuficiente demanda laboral, además, el 69,6\% de ellos manifiesta no conocer el portal de egresados dispuesto por la UNAD, un $78,3 \%$ siente que no ha recibido suficiente apoyo por parte de la oficina de egresados, el $47,8 \%$ no está seguro de que las relaciones actuales de la universidad con las empresas promuevan la ubicación laboral de los egresados y el $46,3 \%$ considera que la reputación de la institución como una entidad que entrega egresados de calidad es media, tal como se puede observar en las figuras 2 a la 5 .

Figura 1.

Ishikawa sobre las relaciones UNAD, Egresados y Empresas

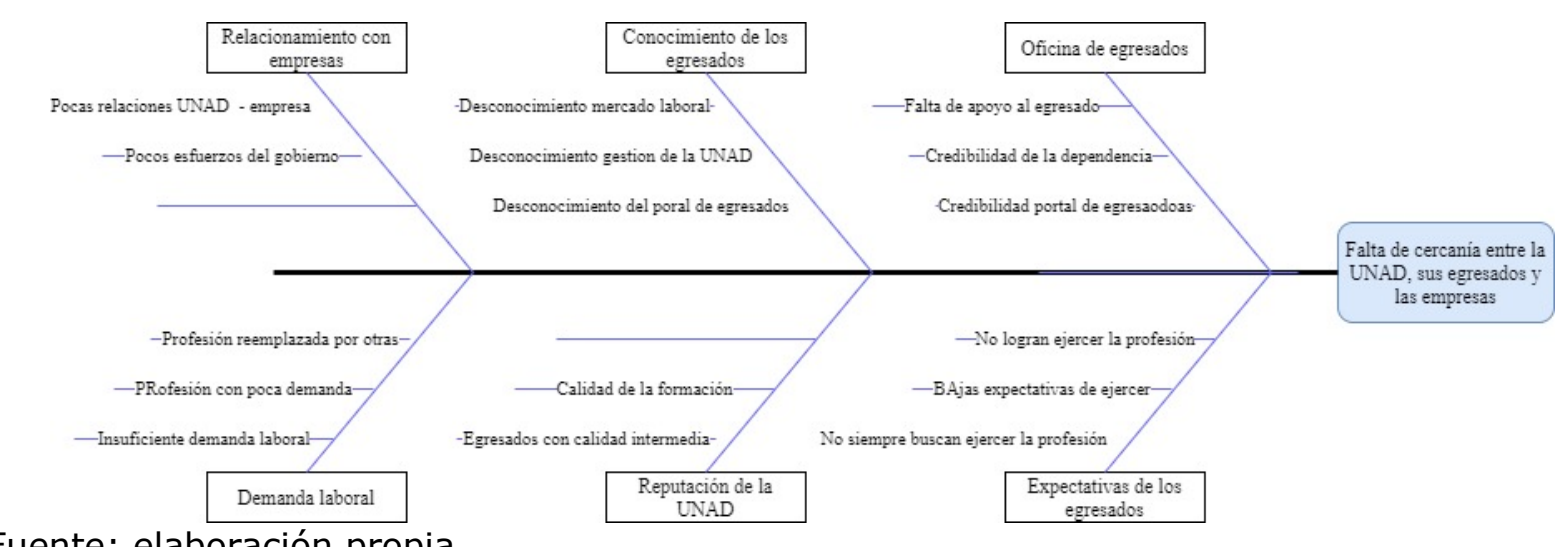

Fuente: elaboración propia

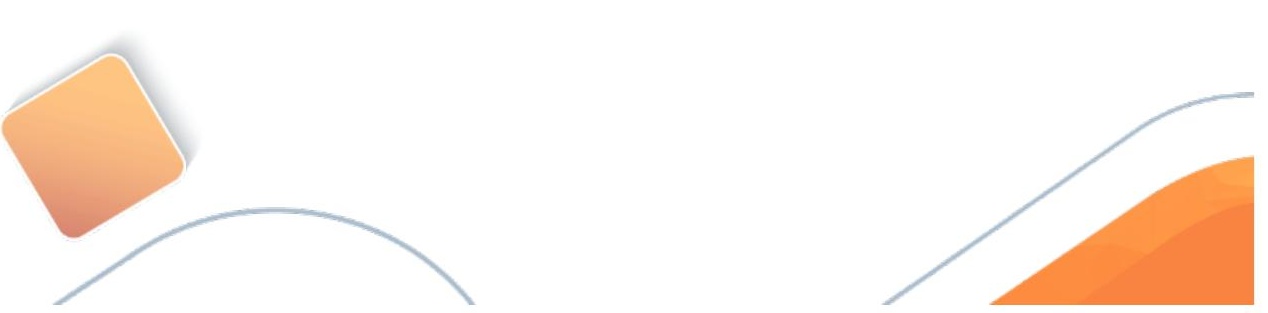


Figura 4.

Reputación de la UNAD ante las empresas

23. ¿Cuál cree usted qué es la reputación de la UNAD en el sector empresarial como institución que entrega egresados de calidad?

322 respuestas

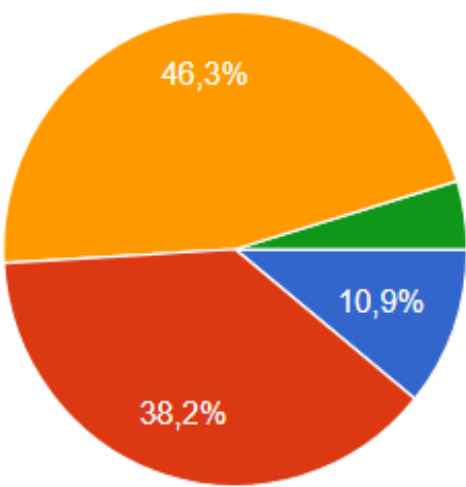

Superior.

Alta.

Media

Baja.

Fuente: elaboración propia

Para brindar mayor claridad sobre las respuestas de los encuestados, se presenta a continuación la Tabla 1 , que contiene varias de las respuestas obtenidas al aplicar el instrumento.

\section{Tabla 1.}

Detalle de las respuestas obtenidas en la encuesta

\section{Respuestas obtenidas en la encuesta.}

El $1.2 \%$ de los egresados considera que la bolsa de empleo de la UNAD constituye una forma fácil para obtener empleo (p. 11)

El 47,8\% de los egresados no se desempeña en la profesión que cursó en la UNAD (p.12)

El $31.1 \%$ no ha logrado ubicarse laboralmente (p. 13)

El $63 \%$ de los egresados busca empleo por razones diferentes a desempeñarse en la profesión que cursó en la UNAD (p. 16)

El $21.1 \%$ de los egresados considera que la profesión cursada no tiene demanda laboral y otro $39,2 \%$ considera que otras profesiones han reemplazado la suya (p.17)

El 40,1\% de los egresados desconoce las tendencias actuales del mercado laboral (p.19)

El $66,8 \%$ de los egresados considera que tiene posibilidades intermedias de ubicarse 


\section{Respuestas obtenidas en la encuesta.}

laboralmente (competitividad intermedia) (p. 20)

El 46,3\% de los egresados considera que la reputación de la UNAD como una institución que entrega egresados de calidad es media (P. 23)

El $66,1 \%$ de los egresados cree que la reputación incide en su fácil ubicación laboral (p.24)

El $92,2 \%$ de los egresados considera necesario que desde la UNAD se instruya a los estudiantes en el uso de las bolsas de empleo (p. 25)

El $54,3 \%$ de los egresados no ha tenido conocimiento de acciones realizadas por la universidad para tratar de impulsar la ubicación laboral de los egresados ( $p .26$ )

El 78,3\% de los egresados no siente que ha recibido un adecuado apoyo de la Oficina de Egresados de la UNAD para su ubicación laboral (p.27)

El 69,6\% de los egresados no conoce el Portal de Egresados de la UNAD (p.28)

El $23,3 \%$ de los egresados no cree que las relaciones actuales de la UNAD con el sector empresarial promueven la ubicación laboral de sus egresados (p.29)

El 47,8\% de los egresados no está seguro de que las relaciones actuales de la UNAD con el sector empresarial promueven la ubicación laboral de sus egresados (p.29)

El 34,8\% de los egresados considera que la gestión de la oficina de egresados de la UNAD en relación con la inserción laboral de los egresados tiene una importancia media, el 19,9\% baja y el $4,3 \%$ dice que no tiene importancia.

El $78,1 \%$ de los egresados considera que la demanda laboral para cubrir la oferta de egresados de las Instituciones de Educación Superior no es suficiente (p. 31)

El $89,8 \%$ de los egresados cree que el gobierno no hace esfuerzos suficientes para impulsar el empleo de los egresados de las Instituciones de educación superior (p.33)

Fuente: Elaboración propia

Ahora, dentro del contexto general sobre el desempleo universitario en Colombia (Rincón y Valencia, 2018), el diseño de un modelo de consultorio empresarial, para el momento en el que se implemente, además de articular a los egresados y contribuir con el fortalecimiento de los procesos estratégicos y organizacionales de las empresas favorecidas (Montoya et. al, 2016), permitirá, también, mejorar la percepción de los egresados con la Universidad y la Oficina de Egresados, pues, desde las experiencias compartidas con la comunidad, esto puede traducirse en una mayor acogida de parte de los estudiantes potenciales para con la Universidad y su intención de acceder a cualquiera de sus programas académicos. 
A medida que los egresados participantes mantengan su continuidad en el ejercicio y sigan teniendo una mayor recepción y aceptación durante el desarrollo de sus actividades, se promoverá su articulación en el sector empresarial con otras organizaciones y se dinamizará la rotación, lo que dará cabida al ingreso de nuevos egresados en la práctica del consultorio.

Los resultados del presente proyecto incentivarán el diseño de nuevas propuestas orientadas a encontrar acciones aplicables desde la universidad para generar nuevas oportunidades de ubicación en el mercado laboral a los egresados, para ello, se constata la necesidad diseñar un modelo de consultorio y, si es el caso, a partir de lo generado, construir desde lo construido.

\section{CONCLUSIONES.}

En este trabajo se fundamentó el diseño de un modelo de consultorio empresarial que dinamice la relación entre la Universidad y el sector productivo, y que genere, como resultado, mayores posibilidades a los egresados para acceder a puestos formales de trabajo, asimismo, la posibilidad de confrontar, en el momento de su implementación, el paradigma de la "inexperiencia" que ralentiza el acceso de los egresados de las IES al mercado laboral.

En el marco del desempleo universitario en Colombia, es importante destacar que, al brindarle a los egresados la posibilidad de articularse en el mercado laboral, se reducen tanto las probabilidades como la temporalidad de permanecer en condición de cesantes, en este sentido, su formación y experiencia no se ven interrumpidas, y se promueve la continuidad del ejercicio profesional en la línea de su formación y calificación mientras acumulan experiencia, pues, como mencionado previamente, en muchas ocasiones, la inexperiencia, lamentablemente, se ha configurado como un escollo al momento de conseguir un trabajo.

Aparte de la fundamentación teórica, ha sido de gran contribución el acceso a los resultados de la investigación realizada durante el año 2020 por el Semillero de Investigación en Desarrollo Sostenible, Gestión Organizacional y Prospectiva (SIDESGOP), perteneciente al grupo de Investigación CANANGUCHALES, considerando que se aplicó una encuesta (previamente validada por pares) a la comunidad de egresados. Esto ha permitido determinar la pertinencia frente al diseño de un consultorio empresarial para los estudiantes y egresados, la escuela ECACEN y el sector productivo. 
